\title{
ON THE ASYMPTOTIC BEHAVIOR OF SOLUTIONS OF A CERTAIN SECOND ORDER ORDINARY DIFFERENTIAL EQUATION
}

\author{
By ATsushi YoshiKawa
}

\section{§1. Introduction.}

Consider the following ordinary differential operator of second order:

$$
P=\partial_{t}^{2}+k^{2}\left(t^{2}+y^{2}\right)+k c .
$$

Here $k$ belongs to the set $\boldsymbol{R}_{+}$of the positive numbers, $c$ to the complex number field $\boldsymbol{C}$, and $(t, y)$ lies in the Euclid plane $\boldsymbol{R}^{2}$.

The purpose of the present article is to give a fundamental pair of the solutions to the equation:

$$
P u=0
$$

with detailed asymptotic properties as $k \rightarrow+\infty$. The novelty we claim here is its derivation as we will roughly sketch immediately after the statement of our Main Theorem (Theorem 1.1) below. Our asymptotic expansions are in fact different from usually given ones (see Nishimoto [5]). We expect that our results will be extended to partial differential operators such as

$$
\partial_{t}^{2}-\partial_{x}^{2}+k^{2}\left(t^{2}+x^{2}+y^{2}\right)+k c .
$$

Details on the latter case will be discussed elsewhere.

Now we explain what will be required in our formulation of asymptotics. Let

$$
T_{\varepsilon}(t, y)=\varepsilon t+\sqrt{t^{2}+y^{2}}, \quad \varepsilon \in\{+,-\},
$$

and denote by $D_{\varepsilon}$ the set of $(t, y, k)$ such that $k>0, T_{\varepsilon}(t, y)>0, y$ running on the real line $\boldsymbol{R}$. Thus, $D_{\varepsilon}$ is the portion of the half space $\boldsymbol{R}_{+}^{3}=\{(t, y, k) ; k>0\}$ obtained by deleting the quarter plane $\{(t, y, k) ; \varepsilon t \leqq 0, y=0, k>0\} . D_{\varepsilon}$ and the quarter space $\boldsymbol{R}_{+} \times \boldsymbol{R} \times \boldsymbol{R}_{+}$are diffeomorphic by the bijection:

$$
\Phi_{\varepsilon}(t, y, k)=\left(T_{\varepsilon}(t, y), y, k\right), \quad(t, y, k) \in D_{\varepsilon} .
$$

We will consider everything in the Fréchet space $\mathcal{E}\left(D_{\varepsilon}\right)$ of the infinitely differen-

Received December 3, 1984 
tiable functions on $D_{\varepsilon}$. Several particular classes of subspaces of $\mathcal{E}\left(D_{\varepsilon}\right)$ will play essential roles in our asymptotic expansions. Denote by $\boldsymbol{R}_{+}$the multiplicative group of the positive numbers. The $\boldsymbol{R}_{+}$-action in $D_{\varepsilon}$ :

$$
g_{\rho}:(t, y, k) \longrightarrow\left(\rho^{-1 / 2} t, \rho^{-1 / 2} y, \rho k\right), \quad \rho>0,
$$

and

$$
h_{\rho}:(t, y, k) \longrightarrow(t, y, \rho k), \quad \rho>0,
$$

respectively induce the differentiable $\boldsymbol{R}_{+}$-action in $\mathcal{E}\left(D_{\varepsilon}\right)$ :

$$
\left(g_{\rho} u\right)(t, y, k)=u\left(g_{\rho}(t, y, k)\right)
$$

and

$$
\left(h_{\rho} u\right)(t, y, k)=u\left(h_{\rho}(t, y, k)\right),
$$

$u \in \mathcal{E}\left(D_{\varepsilon}\right)$ (cf. Yoshikawa [9]).

Let $\mu \in C$. We denote by $\Gamma^{\mu}\left(D_{\varepsilon} ; h_{\rho}\right)$ the set of the $h_{\rho}$-homogeneous elements of degree $\mu$ :

$$
\Gamma^{\mu}\left(D_{\varepsilon} ; h_{\rho}\right)=\left\{u \in \mathcal{E}\left(D_{\varepsilon}\right) ; h_{\rho} u=\rho^{\mu} u \text { for all } \rho>0\right\} .
$$

$\Gamma^{\mu}\left(D_{\varepsilon} ; g_{\rho}\right)$ is defined analogously. On the other hand, for any $s \in \boldsymbol{R}$, we denote by $\mathcal{B}^{s}\left(D_{\varepsilon} ; h_{\rho}\right)$ the totality of those $u \in \mathcal{E}\left(D_{\varepsilon}\right)$ such that for any $\rho_{0}>0$ the set $\left\{\rho^{-s} h_{\rho} u ; \rho \geqq \rho_{0}\right\}$ is bounded in $\mathcal{E}\left(D_{\varepsilon}\right)$. We will also use the spaces

$$
\mathscr{B}^{s+0}\left(D_{\varepsilon} ; h_{\rho}\right)=\bigcap_{r>0} \mathcal{B}^{s+r}\left(D_{\varepsilon} ; h_{\rho}\right)
$$

and

$$
\mathscr{B}^{-\infty}\left(D_{\varepsilon} ; h_{\rho}\right)=\bigcap_{-\infty<s<\infty} \mathscr{B}^{s}\left(D_{\varepsilon} ; h_{\rho}\right) .
$$

Note $T_{\varepsilon}(t, y) \in \Gamma^{-1 / 2}\left(D_{\varepsilon} ; g_{\rho}\right)$. The operator $P$ is $g_{\rho}$-homogeneous of degree 1 in the sense

$$
g_{\rho} P g_{\rho}^{-1}=\rho P, \quad \rho>0 .
$$

However, we take as the symbol $\sigma_{P}(t, y, \tau, \eta)$ of the operator $P$ the function

Let

$$
\sigma_{P}(t, y, \tau, \eta)=-\tau^{2}+t^{2}+y^{2} .
$$

$$
\Lambda_{\varepsilon}^{\delta}=\left\{(t, y, k \tau, k \eta, k) ; \tau=\varepsilon \delta \sqrt{t^{2}+y^{2}}, \eta=\delta y \log \left\{\sqrt{k} T_{\varepsilon}(t, y)\right\},(t, y, k) \in D_{\varepsilon}\right\},
$$

$\varepsilon, \delta \in\{+,-\}$. Here we adopt the convention: $\varepsilon \delta=+$ if $\varepsilon=\delta$, and $\varepsilon \delta=-$ if $\varepsilon \neq \delta$ for $\varepsilon, \delta \in\{+,-\}$. The symbol $\sigma_{P}$ of $P$ vanishes on $\Lambda_{\varepsilon}^{\delta}$. For each $\varepsilon, \delta, \Lambda_{\varepsilon}^{\delta}$ is invariant under the $\boldsymbol{R}_{+}$-actions $g_{\rho}$ and $h_{\rho} . \quad \Lambda_{\varepsilon}^{\delta}$ is interpreted as a Hamilton flow associated to the Hamiltonian $\tau-\varepsilon \delta \sqrt{\bar{t}^{2}+y^{2}}$. Although 


$$
d \tau \wedge d t+d \eta \wedge d y=-\delta d\left(\frac{1}{4} y^{2}\right) \wedge \frac{d k}{k}
$$

along $\Lambda_{\varepsilon}^{\delta}$, we have a generating function:

$$
S_{\varepsilon}^{\delta}(t, y, k)=\frac{\varepsilon \delta}{2} t \sqrt{t^{2}+y^{2}}+\frac{\delta}{2} y^{2} \log \left\{\sqrt{k} T_{\varepsilon}(t, y)\right\}-\frac{\delta}{4} y^{2}
$$

in the sense

$$
\Lambda_{\varepsilon}^{\delta}=\left\{\left(t, y, k \partial_{t} S_{\varepsilon}^{\delta}(t, y, k), k \partial_{y} S_{\varepsilon}^{\delta}(t, y, k), k\right) ;(t, y, k) \in D_{\varepsilon}\right\} .
$$

Note that $k S_{\varepsilon}^{\delta}(t, y, k)$ is a primitive with respect to $t$ of $\varepsilon \delta k \sqrt{t^{2}+y^{2}}$ and is $g_{\rho^{-}}$ homogeneous of degree 0 . The requirement on $g_{\rho}$-homogeneity determines $S_{\varepsilon}^{\delta}$ uniquely from (1.8) and (1.10). By the way, $S_{\varepsilon}^{\delta}(t, 0, k)=(\delta / 2) t^{2}$ since $\varepsilon t>0$ when $y=0$.

Now we are ready for stating our main result :

THEOREM 1.1. Let $\varepsilon \in\{+,-\}$ be fixed. There are a fundamental pair $v_{\varepsilon}^{+}(t, y, k)$ and $v_{\varepsilon}^{-}(t, y, k)$ of the solutions to the equation (1.2) in $\boldsymbol{R}^{2} \times \boldsymbol{R}_{+}$which enjoy the following properties:

(i) $v_{\varepsilon}^{+}(t, y, k)$ and $v_{\varepsilon}^{-}(t, y, k)$ are infinitely differentiable in $(t, y, k) \in \boldsymbol{R}^{2} \times \boldsymbol{R}_{+}$.

(ii) $v_{\varepsilon}^{\delta}(t, y, k) \exp \left\{-\sqrt{-1} S_{\varepsilon}^{\delta}(t, y, k) k\right\} \in \mathcal{E}\left(D_{\varepsilon}\right), \delta \in\{+,-\}$.

(iii) Let

$$
\mu(\delta)=\frac{1}{4}-\delta \frac{\sqrt{-1}}{4} c, \quad \delta \in\{+,-\} .
$$

There are families of functions

$$
u_{\varepsilon, j}^{\delta}(t, y, k) \in \Gamma^{\mu(\delta)}\left(D_{\varepsilon} ; g_{\rho}\right) \cap \Gamma^{-\jmath}\left(D_{\varepsilon} ; h_{\rho}\right),
$$

$j=0,1,2, \cdots$, such that

$$
u_{\varepsilon, 0}^{\delta}(t, y, k)=\left(t^{2}+y^{2}\right)^{-1 / 4} T_{\varepsilon}(t, y)^{\delta \mathcal{N}-1} c / 2
$$

and that

$$
\begin{aligned}
& v_{\varepsilon}^{\delta}(t, y, k) \exp \left\{-\sqrt{-1} S_{\varepsilon}^{\delta}(t, y, k) k\right\}-\sum_{j<N} u_{\varepsilon, j}^{\delta}(t, y, k) \\
& \quad \in \Gamma^{\mu(\delta)}\left(D_{\varepsilon} ; g_{\rho}\right) \cap \mathscr{B}^{-N}\left(D_{\varepsilon} ; g_{\rho}\right)
\end{aligned}
$$

for any positive integer $N$ (See Lemma 2.2 to decipher function spaces).

(iv) The wronskian is given by

$$
v_{\varepsilon}^{+}(t, y, k) \partial_{t} v_{\varepsilon}^{-}(t, y, k)-v_{\varepsilon}^{-}(t, y, k) \partial_{t} v^{+}(t, y, k)=-2 \sqrt{-1} k .
$$

Remark. (1.12) implies $u_{\varepsilon, j}^{\delta}(t, y, k)=u_{\varepsilon, j}^{\delta}(t, y, 1) k^{-\jmath}, k>0$. Note also

$$
u_{\varepsilon, 0}^{\delta}(t, y, k)=\left|\partial_{t} T_{\varepsilon}(t, y)\right|^{1 / 2} T_{\varepsilon}(t, y)^{-2 \mu(\delta)} .
$$


As far as the equation (1.2) is concerned, there might be several proofs to Theorem 1.1. One such proof might be based on Laplace's method by giving an integral representation of a solution of (1.2). In fact, there have been closely related studies by such an approach (Alinhac [1], Sibuya [6], cf. Yoshikawa $[10])$. Another proof would be done by relating solutions of (1.2) to particular confluent hypergeometric functions (e.g., Erdelyi et al. [3]). Namely,

$$
\begin{aligned}
U^{ \pm}(t, y, k)= & \Psi\left(\frac{1}{4} \mp \frac{\sqrt{-1}}{4} c \mp \frac{\sqrt{-1}}{4} k y^{2}, \frac{1}{2} ; \pm \sqrt{-1} k t^{2}\right) \\
& \cdot \exp \left( \pm \sqrt{-1} k t^{2} / 2\right)
\end{aligned}
$$

make up fundamental pair of the solutions of (1.2). Here, for complex $a$, $\Psi(a, 1 / 2 ; z)$ is a confluent hypergeometric function, and

$$
\begin{aligned}
& \Psi\left(a, \frac{1}{2} ; \mp \sqrt{-1} s^{2}\right)=\frac{\Gamma(1 / 2)}{\Gamma(a+1 / 2)}{ }_{1} F_{1}\left(a, \frac{1}{2} ; \mp \sqrt{ }-1 s^{2}\right) \\
& +\frac{\Gamma(-1 / 2)}{\Gamma(a)} e^{\mp \sqrt{-1} \pi / 4}{ }_{1} F_{1}\left(a+\frac{1}{2}, \frac{3}{2} ; \mp \sqrt{-1} s^{2}\right) s
\end{aligned}
$$

are entire functions of $s$ and $a$ (cf. Yoshikawa [8]). However, through these methods, detailed asymptotic properties of the solutions of (1.2) might not be easily obtained.

On the other hand, our present approach is based on classical Frobenius' method (compare, however, with Taylor [7]). It makes visible how each term in the asymptotic expansions (1.14) is determined from the equation (1.2). Thus, determination of $u_{\varepsilon, j}^{\delta}(t, y, k)$ is rather obvious. The only non-trivial part in our approach is to show that there are actually solutions with given asymptotic expansions. We do this essentially following Erdelyi [2] by solving Volterra type integral equations. Here lie some difficulties, though. For we will be working on the infinite interval $(0,+\infty)$ and in the Fréchet space $\Gamma^{0}\left(D_{\varepsilon} ; g_{\rho}\right) \cap \mathscr{B}^{-\infty}\left(D_{\varepsilon} ; h_{\rho}\right)$. This means that the customary successive approximation is not recommended, but, as we will expound below, a variant of the contraction principle will do the work.

Some further remarks are due now. Firstly, note the operator (1.1) is invariant under the reflections $t \rightarrow-t$ or $y \rightarrow-y$. Also $T_{\varepsilon}(t, y)=T_{\varepsilon^{\prime}}\left(-t, y^{\prime}\right), \varepsilon \neq \varepsilon^{\prime}$, $y^{\prime}= \pm y$. Therefore, Theorem 1.1 provides two sets of fundamental pairs of the solutions of (1.2):

and

$$
v_{+}^{\delta}(t, y, k), \quad \delta \in\{+,-\}
$$

$$
v_{-}^{\delta}(t, y, k), \quad \delta \in\{+,-\} .
$$

However, we do not know the explicit form of the transformation matrix $S=$ $\left(S^{\delta \delta^{\prime}}(y, k)\right)$ :

$$
v_{+}^{\delta}(t, y, k)=\sum_{\delta^{\prime} \in 1+,-1} S^{\delta \delta^{\prime}}(y, k) v_{-}^{\tilde{o}^{\prime}}(t, y, k),
$$


$\delta \in\{+,-\}$.

Secondly, our discussions are valid even if we replace $D_{\varepsilon}$ by the set $\widetilde{D}_{\varepsilon}$ of $(t, y, k) \in \boldsymbol{C} \times \boldsymbol{R} \times \boldsymbol{R}_{+}$such that $T_{\varepsilon}(t, y)$ does not touch the non-positive real axis. We then have to replace $\mathcal{E}\left(D_{\varepsilon}\right)$ by the Fréchet space $\mathcal{O}\left(\tilde{D}_{\varepsilon}\right)$ of the smooth functions on $\widetilde{D}_{\varepsilon}$, holomorphic in $t$, infinitely differentiable in $(y, k)$. Details are left to the reader, including complex extensions of $y$ and $k$.

Finally we recall that a result closely related to Theorem 1.1 has played an essential role in constructing parametrices for a certain class of non-strictly hyperbolic partial differential operators (See [1], [10]).

\section{$\S 2$. Formal construction.}

In the present section, we show the formal part of Theorem 1.1. We take $\varepsilon=+$ and omit the reference to $\varepsilon$ in what follows. So we simply write $D$, $T(t, y), v^{ \pm}(t, y, k), S^{\delta}(t, y, k)$, or $u_{j}^{\delta}(t, y, k)$ instead of $D_{+}, T_{+}(t, y), v_{+}^{ \pm}(t, y, k)$, $S_{+}^{\delta}(t, y, k)$ or $u_{+, j}^{\delta}(t, y, k)$.

Let $S^{\delta}(t, y, k), \delta \in\{+,-\}$, be defined by (1.9), $\varepsilon=+$. Then $S^{\delta}(t, y, k) k \in$ $\Gamma^{0}\left(D ; g_{\rho}\right) \cap \mathscr{B}^{1+0}\left(D ; h_{\rho}\right)$. Let

$$
P^{\delta} u=\exp \left\{-\sqrt{-1} S^{\delta}(t, y, k) k\right\} P\left[\exp \left\{\sqrt{-1} S^{\delta}(t, y, k) k\right\} u\right],
$$

$u \in \mathcal{E}(D), \delta \in\{+,-\}$. The operators $P^{\delta}$ are $g_{\rho}$-homogeneous of degree 1 , but

$$
h_{\rho} P^{\delta} h_{\rho}^{-1}=\rho P_{1}^{\delta}+P_{0}^{\delta},
$$

where

$$
P_{1}^{\delta}=\delta 2 k \sqrt{-1} \sqrt{t^{2}+y^{2}} \partial_{t}+\frac{\delta k \sqrt{-1} t}{\sqrt{t^{2}+y^{2}}}+k c
$$

and

$$
P_{0}^{\delta}=\partial_{t}^{2}
$$

The relation (2.2) is a decomposition of the operators $P^{\delta}$ into $h_{\rho}$-homogeneous parts.

Proposition 2.1. There are a pair of formal series

$$
u_{*}^{\delta}(t, y, k)=\sum_{j=0}^{\infty} u_{j}^{\delta}(t, y, k), \quad \delta \in\{+,-\},
$$

such that $v_{*}^{\delta}(t, y, k)=u_{*}^{\delta}(t, y, k) \exp \left\{\sqrt{-1} S^{\delta}(t, y, k) k\right\}, \delta \in\{+,-\}$, are formal solutions of the equation $P v=0$. Furthermore, for $j \geqq 0$,

$$
u_{j}^{\delta}(t, y, k) \in \Gamma^{\mu(\delta)}\left(D ; g_{\rho}\right) \cap \Gamma^{-\jmath}\left(D ; h_{\rho}\right),
$$

where $\mu(\delta)$ are given by (1.11), $\delta \in\{+,-\} . u_{j}^{\delta}(t, y, k)$ are determined from the equations: 


$$
P_{1}^{\delta} u_{0}^{\delta}=0
$$

and

$$
P_{1}^{\delta} u_{\jmath}^{\delta}+P_{0}^{\delta} u_{\jmath-1}^{\delta}=0
$$

for $j \geqq 1$. In particular, we can take

$$
u_{0}^{\delta}(t, y, k)=\left(t^{2}+y^{2}\right)^{-1 / 4} T(t, y)^{\delta \sqrt[v]{-1} c / 2} .
$$

The proof of this proposition is based on two facts. One is the following observation :

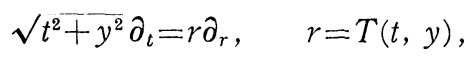

and another is the following

Lemma 2.2. Let $u(t, y, k) \in \Gamma^{\nu}\left(D ; g_{\rho}\right) \cap \mathcal{B}^{s}\left(D ; h_{\rho}\right), \quad s \in \boldsymbol{R}, \nu \in \boldsymbol{C}$. Then for any $k_{0}>0, y_{0}>0, r_{0}>0$ and any integer $\imath, j, m \geqq 0$,

$$
\left|\partial_{l}^{i} \partial_{y}^{j} \partial_{k}^{m} u(t, y, k)\right| \leqq C T(t, y)^{-2 \operatorname{Re} \nu-\imath-\jmath+2 s} k^{s-m}
$$

for $T(t, y) \geqq r_{0},|y| \leqq y_{0} T(t, y), k \geqq k_{0}$, with a positve constant $C$ depending on $k_{0}$, $y_{0}, r_{0}, i, j, m$.

Let us show (2.11) for $i=j=m=0$. Since $u \in \Gamma^{\nu}\left(D ; g_{\rho}\right), u(t, y, k)=$ $r^{-2 \nu} u\left(r^{-1} t, r^{-1} y, r^{2} k\right), r=T(t, y)$. Note $T\left(r^{-1} t, r^{-1} y\right)=1$ then. Since $u \in \mathscr{B}^{s}\left(D ; h_{\rho}\right)$, $\left|u\left(r^{-1} t, r^{-1} y, r^{2} k\right)\right| \leqq C\left(r^{2} k\right)^{s}$ if $r^{2} k \leqq r_{0}^{2} k_{0}$ and $\left|r^{-1} y\right| \leqq y_{0}, T\left(r^{-1} t, r^{-1} y\right)=1$, with an appropriate constant $C>0$.

Returning to the proof of Proposition 2.1, we observe

$$
P_{1}^{\delta}=2 \delta k\left(t^{2}+y^{2}\right)^{-1 / 4} T(t, y)^{(\delta \sqrt{-1} / 2) c} \sqrt{t^{2}+y^{2}} \partial_{t}\left\{\left(t^{2}+y^{2}\right)^{1 / 4} T(t, y)^{-(\delta \sqrt{-1} / 2) c} .\right\} .
$$

(2.9) is then immediate from (2.7). Putting

$$
U_{j}^{\delta}(t, y, k)=u_{j}^{\delta}(t, y, k) / u_{0}^{\delta}(t, y, k), \quad j \geqq 0,
$$

we obtain from (2.8) and (2.10),

$$
r \partial_{r} u_{j}^{\delta}+\delta(2 \sqrt{-1} k)^{-1}\left(u_{0}^{\delta}\right)^{-1} \partial_{t}^{2}\left(u_{0}^{\delta} U_{j-1}^{\delta}\right)=0,
$$

$r=T(t, y)$. Now the induction on $j$ works. In fact, $U_{0}^{\delta}=1$. If $U_{j-1}^{\delta} \in \Gamma^{0}\left(D ; g_{\rho}\right)$ $\cap \Gamma^{-\jmath+1}\left(D ; h_{\rho}\right)$, then

$$
(2 \sqrt{-1} k)^{-1}\left(u_{0}^{\delta}\right)^{-1} \partial_{t}^{2}\left(u_{0}^{\delta} \cdot U_{j-1}^{\delta}\right) \in \Gamma^{0}\left(D ; g_{\rho}\right) \cap \Gamma^{-\jmath}\left(D ; h_{\rho}\right) .
$$

Thus, by Lemma 2.2, we can integrate $(2 \sqrt{-1} k)^{-1} r^{-1} u_{0}^{\delta} \partial_{t}^{2}\left(u_{0}^{\delta} U_{j-1}^{\delta}\right), r=T(t, y)$, from $r=+\infty$ to $r=T(t, y)$ parallel to the $r$-axis in the $(r, y)$-plane. We then have $U_{j}^{\delta} \in \Gamma^{0}\left(D ; g_{\rho}\right) \cap \Gamma^{-\jmath}\left(D ; h_{\rho}\right)$, and the induction is complete. 
PROPOSITION 2.3. There are a pair of functions

$$
u_{a}^{\delta}(t, y, k) \in \Gamma^{\mu(\delta)}\left(D ; g_{\rho}\right) \cap \mathscr{B}^{0}\left(D ; h_{\rho}\right)
$$

such that for each $N=0,1,2, \cdots$,

$$
u_{a}^{\delta}(t, y, k)-\sum_{j=0}^{N} u_{j}^{\delta}(t, y, k) \in \Gamma^{\mu(\delta)}\left(D ; g_{\rho}\right) \cap \mathcal{B}^{-N-1}\left(D ; h_{\rho}\right) .
$$

Here is a standard proof. Let $\phi(\xi) \in C^{\infty}(\boldsymbol{R})$ such that $\phi(\xi)=1$ for $\xi>1$ and $\phi(\xi)=0$ for $\xi<1 / 2$. Let

$$
\phi_{n}(t, y, k)=\phi\left(\rho_{n} T(t, y) \sqrt{k}\right),
$$

$\rho_{n}>0$ to be chosen later. We have

and

$$
\phi_{n} \in \Gamma^{0}\left(D ; g_{\rho}\right) \cap \mathscr{B}^{0}\left(D ; h_{\rho}\right)
$$

$$
1-\phi_{n} \in \Gamma^{0}\left(D ; g_{\rho}\right) \cap \mathscr{B}^{-\infty}\left(D ; h_{\rho}\right) .
$$

Let $K_{m, p}=\left\{(t, y, k) \in D ; m^{-1} \leqq T(t, y) \leqq m,|y| \leqq m T(t, y), 2^{-p} \leqq k \leqq 2^{p}\right\} m=1,2, \cdots$, $p=1,2, \cdots$. Then $K_{m, p}$ are compact and $\cup_{m, p} K_{m, p}=D$. For integers $i, j, l \geqq 0$, let

$$
\Phi_{\imath, \jmath, l}=\sup \left|\xi^{-2 i}\left(\frac{1}{2 \xi} \frac{\partial}{\partial \xi}\right)^{\jmath}\left(\phi^{(l)}(\xi) \xi^{l}\right)\right|
$$

and for integers $i, j, l, N \geqq 0, n, m, p \geqq 1, q=0, \pm 1, \pm 2, \cdots$,

$$
\Psi_{n ; \imath, \jmath, l, m, p, q}=\sup _{K_{m, l}}\left|r^{q} \partial_{r}^{i} \partial_{y}^{j} \partial_{k}^{l} U_{n}^{\delta}(t, y, k)\right|
$$

$(r=T(t, y))$, where $U_{n}^{\delta}(t, y, k)$ are those of (2.13). We now take $\rho_{n}$ as follows. $\rho_{0}$ and $\rho_{1}$ may be arbitrary. For $n \geqq 2$, we take $0<\rho_{n} \leqq \min \left\{1,2^{-n} / A^{n}\right\}$, where

$$
A_{n}=\sup \sum_{i^{\prime} \leqq 2, j^{\prime} \leqq j}\left(\begin{array}{c}
i \\
i^{\prime}
\end{array}\right)\left(\begin{array}{l}
j \\
j^{\prime}
\end{array}\right) \Phi_{J-j^{\prime}+n-N, j^{\prime}, i^{\prime}} \Psi_{n, \imath-i^{\prime}, l, \jmath-j^{\prime}, m, p, 2 \jmath-i^{\prime}+2 n-2 N},
$$

supremum being taken over $i, j, l, N \geqq 0, m, p \geqq 1$ such that $i+j+l+p+m+N+1$ $\leqq n$ (cf. Hörmander [4]). It then follows that the sums $\sum_{n=0}^{\infty} U_{n}^{\delta}(t, y, k) \phi_{n}(t, y, k)$ converges in $\Gamma^{0}\left(D ; g_{\rho}\right) \cap \mathscr{B}^{0}\left(D ; h_{\rho}\right)$. Then

$$
u_{a}^{\delta}(t, y, k)=u_{0}^{\delta}(t, y, k) \sum_{n=0}^{\infty} U_{n}^{\delta}(t, y, k) \phi_{n}(t, y, k)
$$

meet the requirements (2.15) and (2.16).

COROLLARY 2.4. We have

$$
W_{a}^{\delta}(t, y, k)=P^{\delta} u_{a}^{\delta}(t, y, k) \in \Gamma^{1+\mu(\delta)}\left(D ; g_{\rho}\right) \cap \mathscr{B}^{-\infty}\left(D ; h_{\rho}\right),
$$

$\delta \in\{+,-\}$, and 


$$
\begin{aligned}
R_{a}^{\delta}(t, y, k) & =P\left(u_{a}^{\delta}(t, y, k) \exp \left\{\sqrt{-1} S^{\delta}(t, y, k) k\right\}\right) \\
& \in \Gamma^{1+\mu(\delta)}\left(D ; g_{\rho}\right) \cap \mathcal{B}^{-\infty}\left(D ; h_{\rho}\right) .
\end{aligned}
$$

Proof. (2.17) follows from (2.16), (2.12), (2.13), and (2.14). (2.18) is then im mediate from (2.1).

\section{$\S 3$. Exact solutions asymptotic to formal ones.}

Corollary 2.4 means that

$$
v_{a}^{\delta}(t, y, k)=u_{a}^{\delta}(t, y, k) \exp \left\{\sqrt{-1} S^{\delta}(t, y, k) k\right\}, \quad \delta \in\{+,-\},
$$

are approximate solutions of the equation (1.2). We show in this section that we can find complementary functions

$$
z^{\delta}(t, y, k) \in \Gamma^{\mu(\delta)}\left(D ; g_{\rho}\right) \cap \mathcal{B}^{-\infty}\left(D ; h_{\rho}\right)
$$

so that

$$
v^{\delta}(t, y, k)=\left(u_{a}^{\delta}(t, y, k)+z^{\delta}(t, y, k)\right) \exp \left\{\sqrt{-1} S^{\delta}(t, y, k) k\right\}
$$

are exact solutions of the equation (1.2), which admit the asymptotic expansions (1.14).

Let

$$
V_{0}^{\delta}(t, y, k)=u_{0}^{\delta}(t, y, k) \exp \left\{\sqrt{-1} S^{\delta}(t, y, k) k\right\} .
$$

Then $V_{0}^{\delta}(t, y, k) \in \Gamma^{\mu(\delta)}\left(D ; g_{\rho}\right)$ and

$$
\left|V_{0}^{\delta}(t, y, k)\right|>0
$$

Let

$$
Z^{\delta}(t, y, k)=z^{\delta}(t, y, k) / u_{0}^{\delta}(t, y, k) .
$$

We want to determine $Z^{\delta}(t, y, k)$ from

$$
P\left(V_{a}^{\delta}(t, y, k)+V_{0}^{\delta}(t, y, k) Z^{\delta}(t, y, k)\right)=0
$$

with

$$
Z^{\delta}(t, y, k) \in \Gamma^{0}\left(D ; g_{\rho}\right) \cap \mathcal{B}^{-\infty}\left(D ; h_{\rho}\right) .
$$

It will often be more convenient to discuss in the arguments $r=T(t, y), y, k$ rather than in the original ones $t, y, k$. A given function $W(t, y, k)$ will be written $\hat{W}(r, y, k)$ if it is expressed in $r, t, k$. In other words. $\hat{W}(T(t, y), y, k)$ $=W(t, y, k)$.

Proposition 3.1. Let 


$$
\hat{K}^{\delta}\left(r, r^{\prime}, y, k\right)=\frac{r^{\prime 2}+y^{2}}{2 r^{\prime 2}} \int_{r^{\prime}}^{r} \hat{V}_{0}^{\delta}(p, y, k)^{-2} \frac{p^{2}+y^{2}}{2 p^{2}} d p \cdot\left(V_{0}^{\delta} P V_{0}^{\delta}\right)^{\wedge}\left(r^{\prime}, y, k\right) .
$$

Then $\hat{Z}^{\delta}(r, y, k)$ satisfy the integral equatıons:

$$
\hat{Z}^{\delta}(t, y, k)+\int_{+\infty}^{r} \hat{K}^{\delta}\left(r, r^{\prime}, y, k\right) \hat{Z}^{\grave{o}}\left(r^{\prime}, y, k\right) d r^{\prime}=\hat{Q}^{\delta}(r, y, k)
$$

in $\Gamma^{0}\left(D ; g_{\rho}\right) \cap \mathscr{B}^{-\infty}\left(D ; h_{\rho}\right)$. Here

$$
\begin{aligned}
\hat{Q}^{\delta}(r, y, k) & =-\int_{\infty}^{r} \frac{r^{\prime 2}+y^{2}}{2 r^{\prime 2}} \int_{r^{\prime}}^{r} \hat{V}_{0}^{\delta}(p, y, k)^{-2} \frac{p^{2}+y^{2}}{2 p^{2}} d p \cdot \hat{V}_{0}^{\delta}\left(r^{\prime}, y, k\right) R_{a}^{\delta}\left(r^{\prime}, y, k\right) d r^{\prime} \\
& \in \Gamma^{0}\left(D ; g_{\rho}\right) \cap \mathcal{B}^{-\infty}\left(D ; h_{\rho}\right) .
\end{aligned}
$$

Proof. First observe that (3.7) (3.1) and (2.18) imply

$$
\partial_{t}\left[\left(V_{0}^{\delta}\right)^{2} \partial_{t} Z^{\delta}\right]+\left(V_{0}^{\delta} P V_{0}^{\delta}\right) Z^{\delta}+V_{0}^{\delta} R_{a}^{\delta}=0,
$$

or

$$
\frac{2 r^{2}}{r^{2}+y^{2}} \partial_{r}\left[\left(\hat{V}_{0}^{\delta}\right)^{2} \frac{2 r^{2}}{r^{2}+y^{2}} \partial_{r} \hat{Z}^{\delta}\right]+\left(V_{0}^{\delta} P V_{0}^{\delta}\right)^{\wedge} \cdot \hat{Z}^{\delta}+\hat{V}_{0}^{\delta} \hat{R}_{a}^{\delta}=0,
$$

because of (1.3) (2.10). By Lemma 2.2 and (3.8) we have

$$
\left(\hat{V}_{0}^{\delta}\right)^{2} \frac{2 r^{2}}{r^{2}+y^{2}} \partial_{r} \hat{Z}^{\delta}+\int_{+\infty}^{r} \frac{r^{\prime 2}+y^{2}}{2 r^{\prime 2}}\left(V_{0}^{\delta} P V_{0}^{\delta}\right)^{\wedge} \cdot Z^{\delta} d r^{\prime}+\int_{+\infty}^{r} \frac{r^{\prime 2}+y^{2}}{2 r^{\prime 2}} V_{0}^{\delta} R_{a}^{\delta} d r^{\prime}=0,
$$

integrating along a line prallel to the $r$-axis in the $(r, y)$-plane. Integrating once more, and changing the order of integrations, we obtain (3.10). To verify (3.11) as well as for a later purpose we state the following

LEMMA 3.2. For $0<r \leqq r^{\prime}, y \in \boldsymbol{R}, k>0$, we have

$$
\hat{K}^{\delta}\left(\rho^{-1 / 2} r, \rho^{-1 / 2} r^{\prime}, \rho^{-1 / 2} y, \rho k\right)=\rho^{1 / 2} \hat{K}^{\delta}\left(r, r^{\prime}, y, k\right),
$$

$\rho>0, \delta \in\{+,-\}$. For any non-negative integers $i, i^{\prime}, j, l$,

$$
\left|\partial_{r}^{i} \partial_{r^{\prime}}^{i^{\prime}} \partial_{y}^{j} \partial_{k}^{l} \hat{K}^{\delta}\left(r, r^{\prime}, y, k\right)\right| \leqq C k^{\imath+i^{\prime}+\jmath-l}
$$

if $r_{0} \leqq r \leqq r^{\prime} \leqq r_{1},|y| \leqq y_{0}, k \geqq k_{0}$. Here $r_{0}, r_{1}, y_{0}, k_{0}$ are arbitrary positive numbers and $C$ is a positive constant depending on $i, i^{\prime}, j, l, r_{0}, r_{1}, y_{0}, k_{0}$.

Proof. Obvious.

Let

$$
\left(K^{\delta} f\right)(t, y, k)=\left.\int_{\infty}^{r} \hat{K}^{\delta}\left(r, r^{\prime}, y, k\right) \hat{f}\left(r^{\prime}, y, k\right) d r^{\prime}\right|_{r=T(t, y)} .
$$

(3.13) then implies that $K^{\delta} f$ belongs to $\Gamma^{0}\left(D ; g_{\rho}\right) \cap \mathscr{B}^{-\infty}\left(D ; h_{\rho}\right)$ if so does $f$. Using a similar estimate to (3.13), we see (3.11) because of (2.18). This completes the proof of Proposition 3.1. 
Thus to show the existence of $Z^{\delta}(t, y, k)$ with (3.7), (3.8), it is enough to show

PROPOSITION 3.3. The operator $I+K^{\delta}$ in $\Gamma^{0}\left(D ; g_{\rho}\right) \cap \mathcal{B}^{-\infty}\left(D ; h_{\rho}\right)$ is a bijection for each $\delta \in\{+,-\}$. Here $I$ is the identity operator and $K^{\delta}$ the integral operator (3.14).

Before giving a proof of this proposition, we rewrite the space $\Gamma^{0}\left(D ; g_{\rho}\right)$ $\cap \mathscr{B}^{-\infty}\left(D ; h_{\rho}\right)$ in a more convenient form. We also rewrite the integral operator (3.14) accordingly.

We begin by introducing the space $\mathcal{S}\left(\boldsymbol{R}_{+} \times \boldsymbol{R}\right)$ which consists of the functions $p(r, y) \in C^{\infty}\left(\boldsymbol{R}_{+} \times \boldsymbol{R}\right)$ such that for each $r_{0}>0, \quad y_{0}>0$ and non-negative integers $i, j, N$ there is a positive constant $C$ for which

$$
\left|\partial_{r}^{i} \partial_{y}^{\jmath} p(r, y)\right| \leqq C r^{-N}
$$

holds when $r \geqq r_{0},|y| \leqq y_{0} r$. Let $p(r, y) \in \mathcal{S}\left(\boldsymbol{R}_{+} \times \boldsymbol{R}\right)$. We define the mapping $\theta$ by

$$
(\theta p)(t, y, k)=p(\sqrt{k} T(t, y), \sqrt{k} y), \quad p \in \mathcal{S}\left(\boldsymbol{R}_{+} \times \boldsymbol{R}\right) .
$$

Proposition 3.4. The spaces $\Gamma^{0}\left(D ; g_{\rho}\right) \cap \mathscr{B}^{-\infty}\left(D ; h_{\rho}\right)$ and $\mathcal{S}\left(\boldsymbol{R}_{+} \times \boldsymbol{R}\right)$ are isomorphic via the mapping $\theta$.

Proof. $q(t, y, k) \in \mathscr{B}^{-\infty}\left(D, h_{\rho}\right)$ means that for any $y_{0}>0, r_{1}>r_{0}>0, k_{0}>0$ and any non-negative integers $i, j, l, N$, a positive constant $C$ is chosen so that

$$
\left|\partial_{t}^{i} \partial_{y}^{j} \partial_{k}^{l} q(t, y, k)\right| \leqq C k^{-N}
$$

holds when

$$
|y| \leqq y_{0}, \quad r_{0} \leqq T(t, y) \leqq r_{1}, \quad k \geqq k_{0} .
$$

If, furthermore, $q \in \Gamma^{0}\left(D ; g_{\rho}\right)$, then

$$
\begin{aligned}
q(t, y, k) & =q(\sqrt{k} t, \sqrt{k} y, 1) \\
& =q\left(t / T(t, y), y / T(t, y), T(t, y)^{2} k\right) .
\end{aligned}
$$

Now observe that

$$
\sqrt{k} T(t, y) \geqq r_{0}^{\prime}, \quad \sqrt{k}|y| \leqq y_{0}^{\prime} \sqrt{k} T(t, y)
$$

holds under (3.18) if $r_{0}^{\prime}=\sqrt{k_{0}} r_{0}$ and $y_{0}^{\prime}=y_{0} / r_{0}$. Therefore, if $p(r, y)=\hat{q}(r, y, 1)$, then the first equality of (3.19) implies $\theta p=q$ and (3.17) is true if $p \in \mathcal{S}\left(\boldsymbol{R}^{+} \times \boldsymbol{R}\right)$. On the other hand, the second equality of (3.19) and (3.17) imply (3.15) since $T(t / T(t, y), y / T(t, y))=1,|y / T(t, y)| \leqq y_{0}^{\prime}, T(t, y)^{2} k \geqq r_{0}^{\prime 2}$ from (3.20). In particular, $q(t, y, k) \in \Gamma^{0}\left(D ; g_{\rho}\right) \cap \mathcal{B}^{-\infty}\left(D ; h_{\rho}\right)$ if and only if $\hat{q}(r, y, 1) \in \mathcal{S}\left(\boldsymbol{R}_{+} \times \boldsymbol{R}\right)$.

Recall (3.12). Let

$$
K_{\sharp}^{\delta}\left(r, r^{\prime}, y\right)=\hat{K}^{\delta}\left(r, r^{\prime}, y, 1\right), \quad \delta \in\{+,-\} .
$$


LEMMA 3.5. For any $r_{0}>0, y_{0}>0$ and any integers $i, i^{\prime}, j \geqq 0$, there is a posituve constant $C$ such that

$$
\left|\partial_{r}^{i} \partial_{r^{\prime}}^{i^{\prime}} \partial_{y}^{j} K_{\#}^{\delta}\left(r, r^{\prime}, y\right)\right| \leqq C r^{\prime-1+\imath+i^{\prime}+\jmath}\left\{1+\left(r / r^{\prime}\right)^{\delta \operatorname{Im} c+2}\right\}
$$

holds when $r_{0} \leqq r \leqq r^{\prime},|y| \leqq y_{0} r$.

Proof. Since $K^{\delta}\left(r, r^{\prime}, y\right)$ contains exponential factors, the effect of one differentiation is a multiplication by a factor of order 1 with respect to $r^{\prime}$. Thus, it is enough to show (3.22) for $i=i^{\prime}=j=0$. If $\delta \operatorname{lm} c+1 \neq-1$, then from (3.9), (3.21), (2.7), (2.9), (2.13) and (1.3), we have

$$
\left|K_{\#}^{\delta}\left(r, r^{\prime}, y\right)\right| \leqq C\left|\int_{r^{\prime}}^{r} p^{1+\delta \operatorname{Im} c} d p\right| r^{\prime-3-\delta \operatorname{Im} c}
$$

if $r_{0} \leqq r \leqq r^{\prime},|y| \leqq y_{0} r$ and $C$ is an appropriate constant. (3.22) for $i=i^{\prime}=j=0$ is then immediate. If $1+\delta \operatorname{Im} c=-1$, then use the relation:

$$
\frac{p^{2}+y^{2}}{2 p^{2}} \hat{V}_{0}^{\delta}(p, y, 1)^{-2}=\frac{\delta \sqrt{-1}}{2} p^{-\sqrt{-1} \delta c} \partial_{p} \exp \left\{-2 \sqrt{-1} \hat{S}^{\delta}(p, y, 1)\right\},
$$

which is a consequence if (3.4), (2.9) and (1.9). Integrating the integral $\int_{r^{\prime}}^{r} \frac{p^{2}+y^{2}}{2 p^{2}} \hat{V}_{0}^{\delta}(p, y, 1)^{-2} d p$ by parts, we obtain (3.22) for $i=i^{\prime}=j=0$ even when $1+\delta \operatorname{Im} c=-1$.

Now we are ready for proving Proposition 3.3. In view of Proposition 3.4, we show the following

Proposition 3.6. Let

$$
\left(K_{\#}^{\delta} f\right)(r, y)=\int_{+\infty}^{r} K_{\#}^{\delta}\left(r, r^{\prime}, y\right) f\left(r^{\prime}, y\right) d r^{\prime},
$$

$f \in \mathcal{S}\left(\boldsymbol{R}_{+} \times \boldsymbol{R}\right)$. For any $g(r, y) \in \mathcal{S}\left(\boldsymbol{R}_{+} \times \boldsymbol{R}\right)$, [there is a unique $h(r, y) \in \mathcal{S}\left(\boldsymbol{R}_{+} \times \boldsymbol{R}\right)$ such that $h+K_{\#}^{\delta} h=g$.

Proof. We introduce the following auxiliary Banach spaces. Let $r_{0}>0, y_{0}>0$ be arbitrarily given. Let $N$ be a positive integer. We denote by $\mathcal{S}\left(r_{0}, y_{0}, N\right)$ the space of those $f(r, y)$ which are continuous when $r \geqq r_{0},|y| \leqq y_{0} r$, and $r^{N}|f(r, y)|$ are bounded from above when $r \geqq r_{0},|y| \leqq y_{0} r . \mathcal{S}\left(r_{0}, y_{0}, N\right)$ is a Banach space with the norm

$$
\|f\|=\sup \left\{r^{N}|f(r, y)| ; r \geqq r_{0},|y| \leqq y_{0} r\right\} .
$$

(3.22) and (3.23) imply that if $N+2+\delta \operatorname{Im} c>0$ then $K_{\sharp}^{\delta} f \in \mathcal{S}\left(r_{0}, y_{0}, N\right)$ when $f \in \mathcal{S}\left(r_{0}, y_{0}, N\right)$ and

$$
\left\|K_{\#}^{\delta} f\right\| \leqq C\left\{N^{-1}+(N+2+\delta \operatorname{Im} c)^{-1}\right\}\|f\| .
$$

Thus, if $N$ is so large that

$$
C\left\{N^{-1}+(N+2+\gamma \operatorname{Im} c)^{-1}\right\}<1,
$$


then $I+K_{\#}^{\delta}$ is a bijection in $\mathcal{S}\left(r_{0}, y_{0}, N\right)$. Now if $g(r, y) \in \mathcal{S}\left(\boldsymbol{R}_{+} \times \boldsymbol{R}\right)$, then $g(r, y)$ $\in \mathcal{S}\left(r_{0}, y_{0}, N\right)$ for any $r_{0}, y_{0}, N$. Therefore, we have $h(r, y) \in \mathcal{S}\left(r_{0}, y_{0}, N\right)$ solving $\left(I+K_{\sharp}^{\delta}\right) h=g$ when $r \geqq r_{0},|y| \leqq y_{0} r$, and $N$ is large enough. Uniqueness of the solution in each $\mathcal{S}\left(r_{0}, y_{0}, N\right)$ then implies that $h(r, y)$ is actually defined for $(r, y)$ $\in \boldsymbol{R}_{+} \times \boldsymbol{R}$ and $h(r, y) \in \cap \mathcal{S}\left(r_{0}, y_{0}, N\right)$, where $\mathcal{S}\left(r_{0}, y_{0}, N\right) \subset \mathcal{S}\left(r_{0}^{\prime}, y_{0}^{\prime}, N^{\prime}\right)$ by the natural restriction when $r_{0} \leqq r_{0}^{\prime}, y_{0} \leqq y_{0}^{\prime}, N \geqq N^{\prime}$. Using (3.22), we have similar estimates for the drivatives of $h(r, y)$. Thus, we have $h(r, y) \in \mathcal{S}\left(\boldsymbol{R}_{+} \times \boldsymbol{R}\right)$ which solves $\left(I+K_{\#}^{\delta}\right) h=g$.

\section{§4. Completion of the proof of Theorem 1.1.}

We have so far verified the statements (ii) and (iii) of Theorem 1.1. The remaining statements (i) and (iv) are rather obvious. From our discussions in $\S 3, v^{\delta}(t, y, k), \delta \in\{+,-\}$, are $C^{\infty}$ solutions of the equation (1.2) in $D . v^{\delta}(t, y, k)$ are extended to $C^{\infty}$ solutions of (1.2) in $\boldsymbol{R}^{2} \times \boldsymbol{R}_{+}$because of (1.16) by solving the Cauchy problem with data at $t=1$. (Actually $v^{\delta}(t, y, k)$ are extended to entire analytic functions in $t$ ). The statement (iv) is also clear. For $w=v^{+} \partial_{t} v^{-}-v^{-} \partial_{t} v^{+}$ satisfies $\partial_{t} w=0$ so that (1.13) and (1.14) imply $w=-2 \sqrt{-1} k$.

\section{REFERENCES}

[1] Alinhac, S., Solution explicite du problème de Cauchy pour des opérateurs effectivement hyperboliques, Duke Math. J., 45 (1978), 225-258.

[2] ERdelyi, A., Asymptotic Expansions, Dover, New York, (1956).

[3] Erdelyi, A., W., Magnus, F. Oberhettinger, and G. Tricomi, Higher Transcendental Functions, Vol. 1, 2, 3. McGraw Hill, New York (1953).

[4] Hörmander, L., Pseudo-differential operators and hypoelliptic equations, In: Singular Integral Operators, Amer. Math. Soc. Symp. Pure Math. 10 (1966), 138183.

[5] Nishimoto, T., Uniform asymptotic properties of the WKB method, Koda1 Math. J., 4 (1981), 71-81.

[6] Sibuya, Y., Global Theory of a Second Order Linear Ordinary Differential Equations with a Polynomial Coefficient. North Holland Math. Studies 18, American Elsevier, New York (1975).

[7] TAYLOR, W.C., A complete set of asymptotic formulas for the Whittaker function and the Laguerre polynomials, Journals Mathematics and Physics, 18 (1939), 3449. (MIT Studies in Applied Mathematics).

[8] Yoshikawa, A., Construction of a parametrix for the Cauchy Problem of some weakly hyperbolic equation I, Hokkaido Math. J., 6 (1977), 313-344.

[9] Yoshikawa, A., Abstract aspects of asymptotic analysis, J. Math. Soc. Japan 31 (1979), 513-533.

[10] Yoshikawa, A., Parametrices for a class of effectively hyperbolic operators, Comm. in PDE, 5 (1980), 1073-1151.

Department of Mathematics,

HOKKAIDO UNIVERSITY,

SAPPORO, 060 JAPAX
Present address :

Departwent of Applied Sciejce

Kyushu Univesity

HAKOZAKI, FuKUOKA, 812 JAPAX 